\title{
Projection Filter Method Based on State Estimation to Nonlinear Systems
}

\author{
Lijuan Chen \\ School of Geodesy and Geomatics \\ Wuhan University \\ Wuhan, China \\ Jianli Du \\ School of Geodesy and Geomatics \\ Wuhan University \\ Wuhan, China
}

\author{
Jizhang Sang \\ School of Geodesy and Geomatics \\ Wuhan University \\ Wuhan, China \\ Junyu Chen \\ School of Geodesy and Geomatics \\ Wuhan University \\ Wuhan, China
}

\begin{abstract}
For nonlinear systems (NLS), the estimator design is a crucial and important problem. In this paper, projection-filtermethod (PFM) based state estimation approach is proposed to NLS. As the weak solution of stochastic differential model of NLS is denoted by the Kolmogorov's forward equation, this paper presents a new finite-dimensional approximating filter for nonlinear filtering problem. By using the differential geometrical approach to statistical manifold onto which the stochastic partial differential equation of the probability density is projected, a finite-dimensional stochastic ordinary differential equation of the associating parameters is gained. The arithmetic of the projection filter can be predigested in the case of exponential family. Combining with the Bayes rule, the posterior density of the states is obtained By taking an illustrative example, numerical experiment results show that the new state estimator is feasible and has good performance than PF.
\end{abstract}

Keywords-component; projection filter method; stochastic differential model; Kohnogorov's forward equation

\section{INTRODUCTION}

ESTIMATING the state of a stochastic dynamic system from noisy observations is an important problem in engineering. The extensive work on this problem for linear systems was initiated by Kalman and Bucy[1,2]. In the decades since this early work, many important theoretical results for the linear problem have emerged and the linear filter has found wide practical application. However, since most systems are not truly linear, linear filtering theory does not apply directly to most physical systems. In the more general setting of nonlinear systems, filter theory is less developed but has received attention from a number of researchers since the 1960s [3-6] For linear systems with Gaussian inputs, the probability density function of the state conditioned on the measurements is Gaussian. Hence, optimal linear filters need only propagate the conditional mean and covariance that completely describes the density function. However, for nonlinear systems, the conditional density may not have a finite parameterization. General nonlinear filters must there force propagate the entire density function.
In order to make the Kalman filter apply in non-linear system, Bucy,Sunahara and others put forward Extended Kalman Filter(EKF), its basic idea is that nonlinear system change into linear system, But assumption conditions of EKF algorithm are too harsh, EKF is just effective to nonlinear system that approximates linear system in updated interval. so it is difficult to apply many physical systems[7,8], Subsequently, Julier and Uhlmann proposed and developed UKF(Unscented Kalman Filter) method [9,10] , UKF method assume that system noises satisfy Gaussian distribution, it does not require system is an system which approximates linear system. Julier and Simon made UKF method be applied to the vehicle navigation positioning and got a better result than EKF [11]. In 1993, British scholar Gordon and others proposed Particle Filter algorithm, This method is based on the nonparametric sequential Monte-Carlo of Bayesian principles to simulate recursive filter algorithm [12], Its core is that it use a group with weights of random samples (particles) to approximate posterior probability density of the target[8]. But in actual application, The above algorithms exist shortcomings in the estimation precision, the difficulty of control implementation and calculated amount UKF filter and UKF filter have purpose to obtain second moment of probability distribution of system state. But practical distribution of probability may not be normal distribution, all possibilities of system state are clearly to directly solve probability density function of the state.

In this paper, we construct a nonlinear filter that approximates the exact nonlinear filter for systems with continuous nonlinear dynamics and discrete nonlinear observations. Accordingly, the paper represents a departure from current research directions in nonlinear filtering. Rather than pursue enhancements or modifications of the EKF, or explore dual relationships to various non-optimal control algorithms, we investigate a method of approximating the exact nonlinear filtering problem. Note that if the exact nonlinear filtering problem could be solved directly or approximated efficiently, it would be widely used today in various industrial applications. We hope that this paper is a step in that direction. 
The exact nonlinear filter consists of two dynamic equations[13]:

1) A partial differential equation (Kolmogorov's forward equation) that describes how the conditional density evolves between measurements, and

2) A difference equation (Bayes formula) that describes how it is modified by information supplied by new measurements.

To solve these equations we employ Projection filter method(PFM), a classic procedure for approximating solutions of partial differential equations (PDEs).In the context of the forward Kolmogorov equation, Projection filter method was suggested by Risker's as a possible way to approximate the solution. However, a detailed analysis was not pursued.

Projection filter method assumes that the exact solution to a PDE can be expanded as an infinite sum of basis elements. An approximate solution is found by truncating this sum and projecting the resulting error onto the finite subspace spanned by the basis elements used to approximate the solution.\}b To distinguish the approximate filter from the exact filter, and for lack of a better name, we will refer to the resulting filter as the nonlinear projection filter (NPF).

Using a complex exponential basis as approximating elements, we show that the nonlinear filter can be implemented efficiently (for low-order systems) using discrete cosine transforms (DCT) resulting in a fast nonlinear filter that could be implemented in real time on a digital signal processor. Sinusoidal bases have been used before to implement PFM based Bayes algorithms, but in much different contexts[14].

This work is also a natural extension of the application of Projection filter method to optimal and robust control. While there have been numerous studies that have applied numerical methods for solving PDEs, we are not aware of a careful study of PFM spectral method to the nonlinear filtering problem.

An important issue concerns the convergence of the PFM approximation. We shall prove that the approximation residual converges to zero as the dimension of the finite dimensional subspace used in the approximation tends to infinity. In other words, the NPF converges to the exact nonlinear filter. One limitation in our convergence result is that we do not obtain an explicit estimate of the approximation error. We only show that the approximation error can be made arbitrarily small by making the order of the approximation large enough.

\section{STOCHASTIC DifFERENTIAL MODEL}

Most physical systems evolve continuously in time while measurements may only be taken periodically at discrete time instants. Suppose the n-dimensional state ${ }_{x_{t}}$ of a continuous nonlinear stochastic dynamic system satisfies

$$
d x_{t}=f\left(x_{t}, t\right) d t+g\left(x_{t}, t\right) d \beta_{t} \quad t \geq t_{0}
$$

where $\left\{\beta_{t}, t \geq t_{0}\right\}$ is a p-dimensional Brownian motion with covariance matrix $Q(t) d t$. Let m-dimensional noisy measurements be made at discrete times $t_{k}$

$$
y_{k}=h\left(x_{t_{k}}, t_{k}\right)+v_{k}
$$

where $\left\{v_{k}, k \geq 1\right\}$ is an m-dimensional white Gaussian sequence independent of $d \beta_{t}$ with covariance matrix $R_{k}$. Define the collection of measurements taken up to and including time $t$ as $Y_{k}=\left\{y_{k}, t_{k} \leq t\right\}$. We seek equations of evolution for the conditional density $p\left(x, t \mid Y_{t}\right)$ because it summarizes all the statistical information about the state contained in the measurements $Y_{t}$ and the initial condition $p\left(x, t_{0}\right)$. From $p\left(x, t \mid Y_{t}\right)$,the conditional mean and variance can be computed, which for nonlinear systems generally depend on all of the higher order moments.

$$
\text { Between observations at } t_{k} \text { and } t_{k+1}, p \equiv p\left(x, t \mid Y_{t}\right)
$$

diffuses according to Kolmogorov's forward equation[3].

$$
\frac{\partial p}{\partial t}=\frac{1}{2} \sum_{i, j=1}^{n} \frac{\partial^{2}\left(p\left(g Q g^{\mathrm{T}}\right)_{i j}\right)}{\partial x_{i} \partial x_{j}}-\sum_{i=1}^{n} \frac{\partial\left(f_{i} \cdot p\right)}{\partial x_{i}}
$$

where either $p\left(x, t_{0}\right)$ or $p\left(x, t \mid Y_{t_{k}}\right)$, the measurement update at $t_{k}$, is used as the initial condition, and where $\left(g Q g^{\mathrm{T}}\right)_{i j}$, is the $(i, j)$ th element of the matrix $g Q g^{\mathrm{T}}$. At an observation, $p$ satisfies the Bayes formula

$$
p\left(x, t_{k+1} / Y_{t_{k+1}}\right)=\frac{p\left(y_{k+1} / x\right) p\left(x, t_{k+1}^{-} / Y_{t_{k}}\right)}{\int_{\Omega} p\left(y_{k+1} / x\right) p\left(\xi, t_{k+1}^{-} / Y_{t_{k}}\right) d \xi}
$$

where

$$
\begin{aligned}
& p\left(y_{\mathrm{k}+1} / x\right) \\
& =\frac{\exp \left\{-\frac{1}{2}\left(y_{k}-h\left(x, t_{k}\right)\right)^{T} R_{k}^{-1}\left(y_{k}-h\left(x, t_{k}\right)\right)\right\}}{\sqrt{(2 \pi)^{m} \operatorname{det} R_{k}}}
\end{aligned}
$$

and $t^{-}{ }_{k+1}$ is the instant in time right before the $(k+1)$ sample. For a detailed derivation and discussion of these results, see Ref. 3.

Equations (3) represent dynamic equations for the exact nonlinear filter. Equation (3) is used to compute predictions between measurements, while measurements are used to update the information about the state via (4). Kolmogorov's forward 
equation (3) and its boundary conditions $p\left(x, t_{0}\right)$ have formed a differential equations boundary value problem.

\section{PREDICTION EQUATION}

To apply projection filter method, we first assume that the solution of (3) satisfies

$$
p_{\text {real }}=\sum_{k=0}^{\infty} H_{2 k}(t) X_{k}(x) \equiv H(t) X(x)
$$

where equality is in the sense of the $L_{2}$ norm, and where $\left\{X_{k}\right\}_{k=0}^{\infty}$ is a complete set of basis functions for $L_{2}$. We approximate $p$ by truncating the sum

$$
p=\sum_{k=0}^{N-1} H_{2 k}(t) X_{k}(x)
$$

where the coefficients $H_{2 k}, X_{k}$ are hermit function and satisfy the projection equation

$$
\int_{0}^{\infty} \mathrm{H}_{2 i}(\xi) \mathrm{H}_{2 l}(\xi) w(\xi) d \xi=\left\{\begin{array}{l}
0 \quad i \neq l \\
(2 i) ! 2^{2 i-1} \sqrt{\pi} \quad i=l
\end{array}\right.
$$

if $g, Q, f$ have nothing to do with the time $t$,

$g Q(t) g^{\mathrm{T}} \equiv 2 a, a$ is a constant.so $f=\sum_{k=1}^{N-1} f_{k} H_{k}(x)$

where $\left\{f_{k}\right\}_{k=1}^{N-1}$ is a constant sequence, so

$$
\begin{aligned}
& f_{k}=\frac{1}{2^{k} k ! \sqrt{\pi}} \int_{-\infty}^{\infty} e^{-\xi^{2}} f(\xi) H_{k}(\xi) d \xi \\
& =\frac{-1}{2^{k} k ! \sqrt{\pi}} \int_{-\infty}^{+\infty} f(\xi) d\left(e^{-\xi^{2}} H_{k-1}(\xi)\right) \\
& =\frac{1}{2^{k} k ! \sqrt{\pi}} \int_{-\infty}^{+\infty} f(\xi) e^{-\xi^{2}} H_{k-1}(\xi) d \xi \\
& \ldots \ldots \\
& =\frac{1}{2^{k} k ! \sqrt{\pi}} \int_{-\infty}^{+\infty} f(\xi) e^{-\xi^{2}} H_{0}(\xi) d \xi
\end{aligned}
$$

So

$$
f^{\prime}=\sum_{k=1}^{N-1} f_{k} X_{k}^{\prime}(x)
$$

By Equations (3), we have

$$
\begin{aligned}
& H^{\prime}(t) X(x) \\
& =a H(t) X^{\prime \prime}(x)-2 f_{k} H(t) X^{\prime}(x) X(x)
\end{aligned}
$$

Assuming that

$$
\frac{H^{\prime}(t)}{H(t)}=\frac{a X^{\prime \prime}(x)-2 f_{k} X^{\prime}(x) X(x)}{X(x)}=-\lambda
$$

so

$$
\left\{\begin{array}{l}
H^{\prime}(t)+\lambda H(t)=0 \\
a X^{\prime \prime}(x)-2 f_{k} X^{\prime}(x) X(x)+\lambda X(x)=0
\end{array}\right.
$$

By (11), we have

$$
\begin{gathered}
\left\langle H(t), H_{2 k}(t)\right\rangle \\
=\sum_{i=0}^{\infty} \int H_{2 i}(t) H_{2 k}(t) w(t) d t \\
=(2 k) ! 2^{2 k-1} \sqrt{\pi} \\
\left\langle H^{\prime}(t), H_{2 k}(t)\right\rangle \\
=\sum_{i=0}^{\infty} 8 i(2 i-1) \int H_{2(i-1)}(t) H_{2 k}(t) w(t) d t \\
=8(k+1)(2 k+1)(2 k) ! 2^{2 k-1} \sqrt{\pi} \Delta \mathrm{T}
\end{gathered}
$$

By the Equations $\left\langle H^{\prime}(t)+\lambda H(t), H_{2 k}(t)\right\rangle=0$, we have

$$
\lambda=8(k+1)(2 k+1) \Delta \mathrm{T} \equiv \lambda_{k}
$$

where $\Delta \mathrm{T}$ shows the total time of program is running.so

$$
\begin{aligned}
& a X^{\prime \prime}(x)-2 f_{k} X^{\prime}(x) X(x)+\lambda_{k} X(x)=0 \\
& X(x) \text { denote } X(x)=\sum_{k=0}^{N} c_{k} H_{k}(x), \text { by the above }
\end{aligned}
$$
equation,we have

$$
\begin{aligned}
& \sum_{k=0}^{N}\left[4 a(k+2)(k+1) c_{k+2}-\left(2(k+1) f_{k}-\lambda_{k}\right) c_{k}\right] H_{k}(x) \\
& =0 \\
& \quad \text { So }
\end{aligned}
$$

$$
c_{k+2}=\frac{\left(2(k+1) f_{k}-\lambda_{k}\right)}{4 a(k+2)(k+1)} c_{k} \equiv b_{k+2} c_{k}
$$

where $\left(2(k+1) f_{k}-\lambda_{k}\right) /(4 a(k+2)(k+1)) \equiv b_{k+2}$, $k=0,1, \ldots$,we have

$$
\begin{gathered}
c_{2 k}=\prod_{i=0}^{k} b_{2 i} c_{0} \\
c_{2 k+1}=\prod_{i=0}^{k} b_{2 i+1} c_{1}
\end{gathered}
$$

The solution of the equation is 


$$
\begin{aligned}
& X(x)=\sum_{k=0}^{N} c_{2 k} x^{2 k}+\sum_{k=0}^{N} c_{2 k+1} x^{2 k+1} \\
& =\sum_{k=0}^{N} c_{0} \prod_{i=0}^{\left[\frac{N}{2}\right]} b_{2 i} H_{2 k}(x)+\sum_{k=0}^{N} c_{1} \prod_{i=0}^{\left[\frac{N}{2}-1\right]} b_{2 i+1} H_{2 k+1}(x)
\end{aligned}
$$

where $c_{0}, c_{1}$ are obtained by $p\left(x, t_{0}\right)$,we have

$$
\begin{aligned}
& c_{0}=\frac{2}{l} \int_{-l}^{l} p\left(x, t_{k} / Y_{t_{\mathrm{k}}}\right) H_{k}(x) d x \\
& c_{1}=\frac{2}{l} \int_{-l}^{l} p\left(x, t_{k} / Y_{t_{\mathrm{k}}}\right)^{\prime} H_{k}(x) d x
\end{aligned}
$$

The expression of prior probability density is

$$
\begin{aligned}
& p_{N}\left(x, t \mid z_{t}\right)= \\
& \left(\sum_{k=0}^{N} c_{0} \prod_{i=0}^{\left[\frac{N}{2}\right]} b_{i} H_{2 k}(x)+\sum_{k=0}^{N} c_{1} \prod_{i=0}^{\left[\frac{N}{2}\right]} b_{i} H_{2 k+1}(x)\right) \sum_{j=0}^{N-1} H_{2 j}(t)
\end{aligned}
$$

By the bayesian formula, we can get the posteriori probability density $p\left(x, t_{k+1} / Y_{t_{k+1}}\right)$, so we have conditions ${ }^{\text {mean }} \mathrm{E}\left(x / Y_{t_{\mathrm{k}+1}}\right) \equiv \mathrm{E}_{\mathrm{k}+1}$ and covariance $\sigma\left(x / Y_{t_{\mathrm{k}+1}}\right) \equiv \sigma_{\mathrm{k}+1}$

$$
\mathrm{E}_{\mathrm{k}+1}=\int \cdots \int_{\Omega} \xi p\left(\xi, t_{k+1} / Y_{t_{\mathrm{k}+1}}\right) d \xi_{1} \cdots d \xi_{n}
$$

$\sigma_{\mathrm{k}+1}=$

$$
\iint_{\Omega}\left(\xi-\mathrm{E}\left(\xi / Y_{t_{\mathrm{k}+1}}\right)\right)^{2} p\left(\xi, t_{k+1} / Y_{t_{\mathrm{k}+1}}\right) d \xi_{1} \cdots d \xi_{n}
$$

\section{Prepare Your Paper Before Styling}

In this section, consider a reference system in a nonlinear state space given by [15], which is described as follows:

$$
\begin{gathered}
\dot{X}=A X+W \\
Z_{k}=h\left(X_{k}\right)+e_{k}
\end{gathered}
$$

where

$$
X=\left(x, y, v_{x}, v_{y}, a_{x}, a_{y}\right)^{T} \text { is state vector, }
$$

$R=\operatorname{diag}\left(1,1 \times 10^{-3}\right)^{\prime} Q=\left(1,0,10,1,1,1 \times 10^{-3}, 1 \times 10^{-4}\right)$

, Initial state satisfies normal distribution $P_{0}$, Experimental results are shown in Figure 1 and Figure 11

$$
\begin{aligned}
& P_{0}=\operatorname{diag}\left[1 \mathrm{~m} / \mathrm{s}, 1 \mathrm{~m}, 1 \mathrm{~m} / \mathrm{s}, 1 \mathrm{~m} / \mathrm{s}, 0.1 \mathrm{~m} / \mathrm{s}^{2}, 0.1 \mathrm{~m} / \mathrm{s}^{2}\right] \\
& A=\left(\begin{array}{llllll}
1 & 0 & t & 0 & 0 & 0 \\
0 & 1 & 0 & t & 0 & 0 \\
0 & 0 & 1 & 0 & t & 0 \\
0 & 0 & 0 & 1 & 0 & t \\
0 & 0 & 0 & 0 & t^{2} & 0 \\
0 & 0 & 0 & 0 & 0 & t^{2}
\end{array}\right), h\left(X_{k}\right)=\left[\begin{array}{c}
\frac{x}{\sqrt{x^{2}+y^{2}}} \\
\frac{y}{\sqrt{x^{2}+y^{2}}}
\end{array}\right]
\end{aligned}
$$

Here, for PFM, the interval of time is chosen as $1 \mathrm{~s}$, the quantity of grid is chosen as 50, and the initial state is $\left[50 \mathrm{~km}, 100 \mathrm{~km}, 1 \mathrm{~m} / \mathrm{s}, 1 \mathrm{~m} / \mathrm{s}, 0.3 \mathrm{~m} / \mathrm{s}^{2}, 0.4 \mathrm{~m} / \mathrm{s}^{2}\right]$.For $\mathrm{PF}$, the number of particles is chosen as 500 . the performance output of PFM is shown in Figures 1, 11 and 111. The conditional density functions are plotted with discrete times. After a few measurements, the predicted density function is bimodal with modes approximately centered at plus and minus the absolute value of the actual state, which is what we expect. Numerical experiment results show that the feasibility of the proposed stochastic estimating method based on PFM is confirmed.

The true state trajectory and the conditional means of the trajectories executed by PFM and PF are given in Figures 1 and 11. Obviously for a nonlinear non-Gaussian system, PF estimating output will lose stableness after a few measurement updates. Because the measurement function of PF should be linearized with the current mean estimate at each measuring step and the second moment of probability distribution of system state also has to be obtained, but the real distribution of probability may not be normal distribution, the actual state is outside of the region where the linearization is valid. e result is that the PF estimate jumps outside of the region of attraction. Meanwhile, the PFM performs reasonably well by giving the limited information available from the measurements and does not fail because of the nonlinearities in the system and measurement characteristics.

Figure 1 shows results of estimated mean and root mean square error of the system state, Figure 11 and Figure 111, we notice that the estimated means and standard deviations of the system state using PFM and particle filter are very similar, The precision speed and position of the PFM increase with time and its high estimated precision and briefness satisfy the need of system state.

\section{CONCLUSION}

An PFM-based state estimation approach to nonlinear systems has been investigated in this paper. It was shown that the new filter can be converged to the true density function in a n-dimensional state space. Furthermore, a detailed demonstration for how to use the filter efficiently is demonstrated, and with a simple example, we can find that PFM outperforms the UKF and the PF reasonably It is envisioned that the filter could be used to process data from sensors with severely nonlinear output characteristics, and how 
to implement the algorithm in parallel, especially for solving high dimension problem. These considerations should be further extended in our future work.

\section{ACKNOWLEDGMENT}

This work was supported by the National Natural science Foundation of China (No.40474024) and the Fundamental Research Funds for the Central Universities of China (No.2042015kf0012).

\section{REFERENCES}

[1] Kalman, R. E., "A New Approach to Linear Filtering and Prediction Problems," Transactions of the ASME, Journal of Basic Engineering, Vol. 82, No. 1, 1960, pp. 34-35.

[2] Kalman R. E., and Bucy, R. S., "New Results in Linear Filtering and Prediction Theory," Transaction of the ASME, Journal of Basic Engineering, M4. 83, 1961, pp.95-108.

[3] Jazwinski, A. H., Stochastic Processes and Filtering Theory, Vol. 64, Mathematics in Science and Engineering, Academic Press, New York, 1970.

[4] Kushner, H. J., "Dynamical Equations for Optimal Nonlinear Filter," Journal ofDifferential Equations, Vol. 3, No. 2, 1967, pp. 179-190.

[5] Kushner, H. J., "Nonlinear Filtering: The Exact Dynamical Equations S atisfied by the Conditional Mode," IEEE Transactions on Autorreatic Controt, Vol. 12, 1967, pp. 262-267.

[6] Stratonovich, R. L., "Conditional Markov Processes," Theory of Probability and Its Applications, Vol. 5, 1960, pp. 156-178.

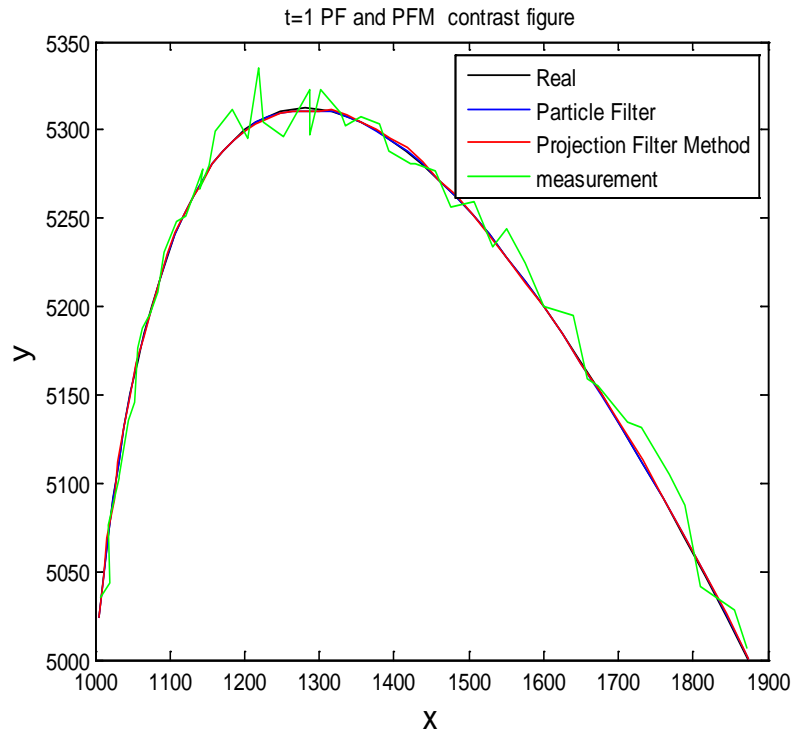

[7] Moylan, P. J, "Note on Kalman-Bucy filters with zero measurement noise," IEEE Transactions on Automatic Control, vol. 19(3), pp.263 $264,1974$.

[8] Johan Bijker, Willem Steyn, "Kalman filter configurations for a lowcost loosely integrated inertial navigation system on an airship," Control Engineering Practice, vol. 16(12), pp.1509 - 1518,2008.

[9] S.J. Julier, J.K, "Uhlmann. non-divergent estimation algorithm in the presence of unknown correlations," Proceedings of the IEEE American Control Conference, Albuquerque, NM, USA, 1997,vol.4, pp. 2369 2373.

[10] Rambabu Kandepu, Bjarne Foss, Lars Imsland, "Applying the unscented Kalman filter for nonlinear state estimation,” Journal of Process Control, vol. 18(7-8), 2008., pp. $753-768$.

[11] Julier, Simon, H.F Durrant-Whyte, "Process models for the high-speed navigation of road vehicles," Proceedings - IEEE International Conference on Robotics and Automation, 1995, vol.1,pp.101 - 105.

[12] Gordon N.J., Salmond D.J., Smith A.F.M., "Novel approach to nonlinearnon-Gaussian Bayesian state estimation,” IEE Proceedings,Part F: Radar and Signal Processing,1993, vol.140(2),pp.107 - 113.

[13] Zhang D S,Wei GW, Kouri D J, "Numerical method for the nonlinear Fokker-Planck equation,” Physical Review E, 1997.vol.56(1),pp.1197 1206.

[14] Yuxin Zhao,Lijuan Chen,Yan Ma.An FEM-based State Estimation Approach to Nonlinear Hybrid Positioning Systems.Mathematical Problems in Engineering. Vol.2013, 2013.

[15] Bucy R S,Senne K D. Digital synthesis of nonlinear filters. Automatica, 1971,vol.7,pp.287-298.

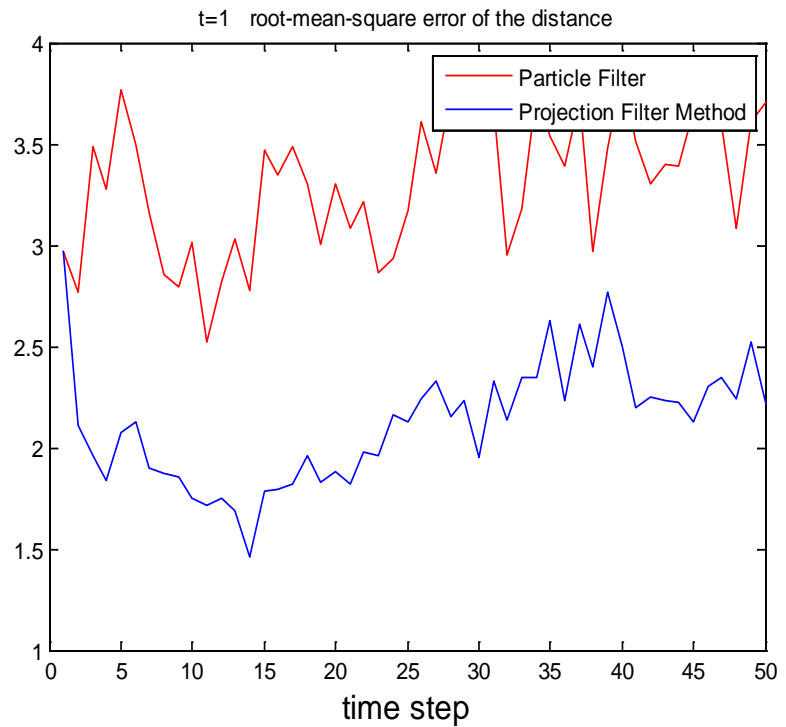

FIGURE I. REAL STATE AND ESTIMATED STATE TRAJECTORIEES BY PFM AND PF 

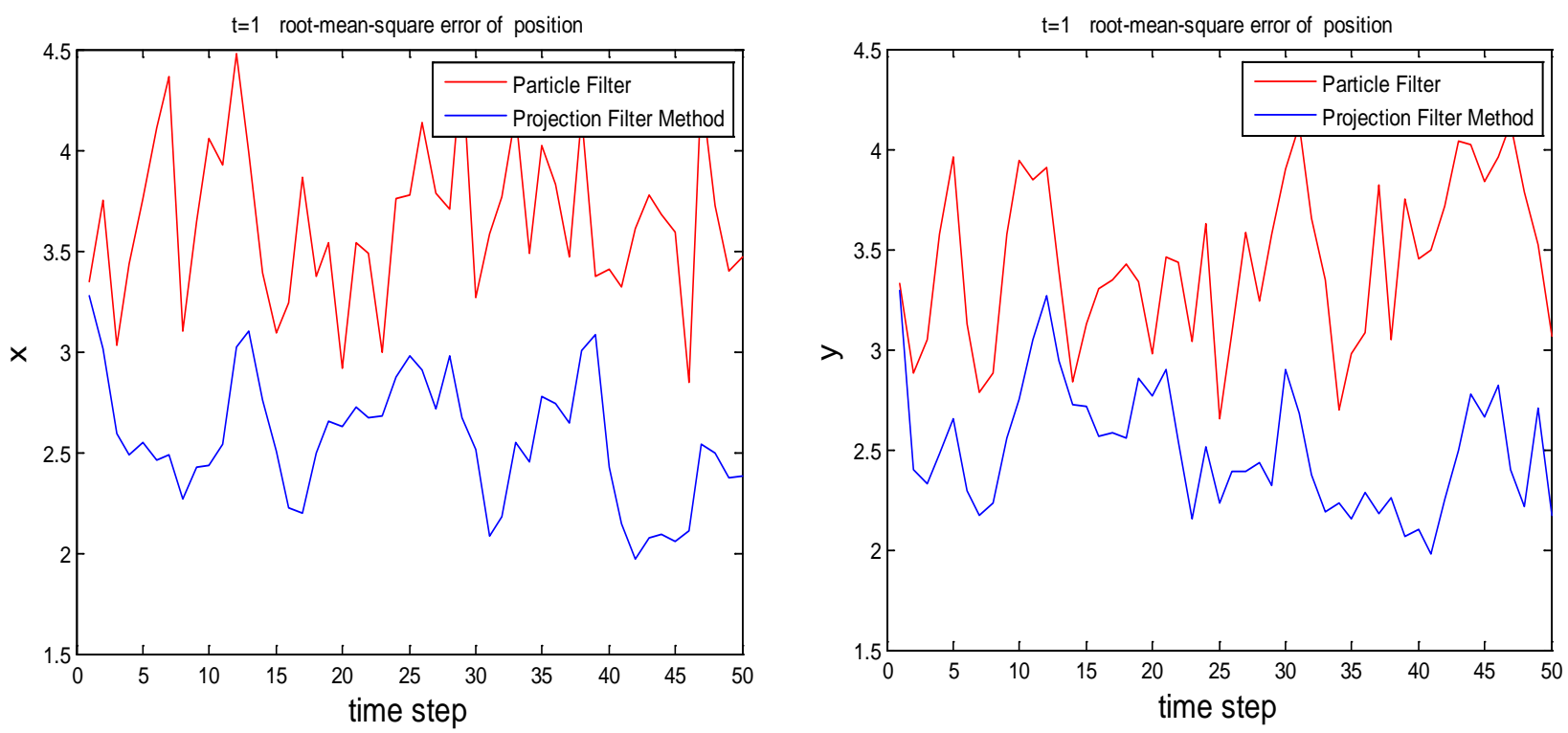

FIGURE II. THE ESTIMATEED MEAN AND ROOT MEAN SQUARE ERROR OF POSITION AND VELOCITY.
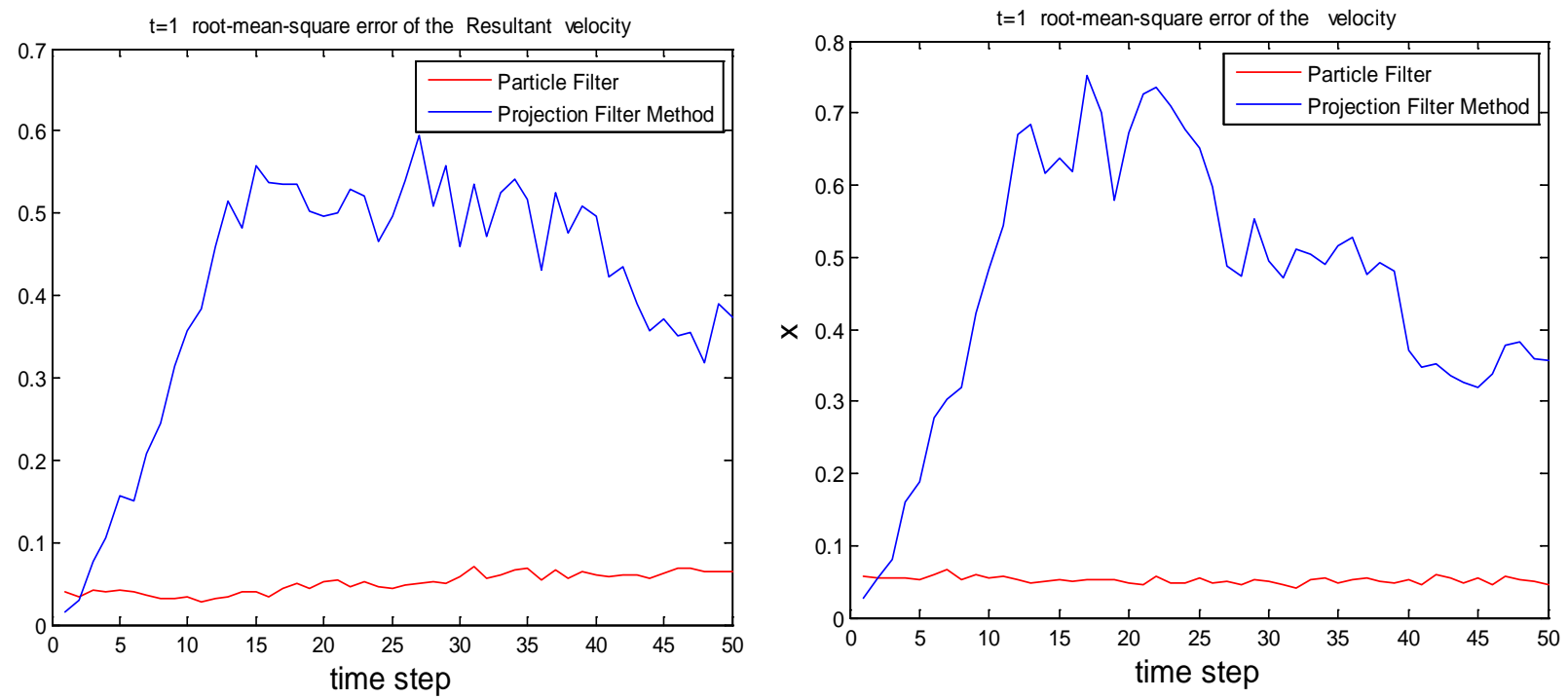

FIGURE III. THE ESTIMATEED MEAN AND ROOT MEAN SQUARE ERROR OF POSITION AND VELOCITY 